Europhysics Letters

PREPRINT

\title{
On random graphs and the statistical mechanics of gran- ular matter
}

\author{
Johannes Berg ${ }^{1}$ And Anita Mehta ${ }^{2}$ \\ 1 Abdus Salam International Centre for Theoretical Physics, 34100 Trieste, Italy \\ 2 S N Bose National Centre for Basic Sciences, Block JD Sector III, Salt Lake, Calcutta \\ 700 098, India
}

PACS. 05.20.-y - Classical statistical mechanics.

PACS. 45.70.Cc - Static sandpiles; granular compaction.

PACS. 75.10.Nr - Spin-glass and other random models.

\begin{abstract}
The dynamics of spins on a random graph with ferromagnetic three-spin interactions is used to model the compaction of granular matter under a series of taps. Taps are modelled as the random flipping of a small fraction of the spins followed by a quench at zero temperature. We find that the density approached during a logarithmically slow compaction - the random-close-packing density - corresponds to a dynamical phase transition. We discuss the role of cascades of successive spin-flips in this model and link them with density-noise power fluctuations observed in recent experiments.
\end{abstract}

Analogies with glasses and spin glasses have long [1] been made to explain the complex properties of granular media, but it was not until the seminal experiments of the Chicago group [2] on granular compaction were carried out that serious theoretical attempts were made to quantify such analogies. These fall into roughly two classes: lattice-based models [3, 4, 8] in a finite-dimensional space (which in general do not admit analytic solutions) or mean field models [9, 10] (where each site interacts with a large number of other sites). In this Letter we argue that models on random graphs may be used to describe those aspects of the behaviour of granular matter which depend on the finite connectivity of the (disordered) grains, while still remaining analytically accessible. In particular this approach allows us to relate phenomena in the compaction of granular matter, such as the random-close-packing density, which is reached asymptotically after many taps, to the structure of the free-energy landscape of spin models on random graphs.

The concept of geometric frustration is thought to be a crucial feature of the behaviour of granular matter. It refers to the existence of voids in the granulate, which cannot be filled in by neighbouring particles due to geometric constraints, either on the mobility of these particles, or on their compatibility in shape or size with the void. A class of models of this feature restrict the number of occupied neighbouring sites of a particle on a lattice, either dynamically [5] or statically [6]. More generally, and expressed in the language of interacting spins (e.g. spin up for the occupied site of a lattice gas model), geometrical frustration may be

(C) EDP Sciences 
written in terms of ferromagnetic nearest-neighbour interactions and anti-ferromagnetic nextnearest neighbour interactions [7]. Models on random graphs may be viewed as the Betheapproximation to finite-dimensional models of this type. The common feature of these models when translated into spin-language is the existence of multi-spin interactions on plaquettes on a random graph 12].

In the following we consider the simplest possible case of a multi-spin model defined on the plaquettes of a random graph, a 3-spin Hamiltonian on a random graph where $N$ binary spins $S_{i}= \pm 1$ interact in triplets

$$
H=-\rho N=-\sum_{i<j<k} C_{i j k} S_{i} S_{j} S_{k}
$$

where the variable $C_{i j k}=1$ with $i<j<k$ denotes the presence of a plaquette connecting sites $i, j, k$ and $C_{i j k}=0$ denotes its absence. Choosing $C_{i j k}=1(0)$ randomly with probability $2 c / N^{2}\left(1-2 c / N^{2}\right)$ results in a random graph, where the number of plaquettes connected to a site is distributed with a Poisson distribution of average $c$. The connection with granular compaction is made in accordance with Edwards' thermodynamic hypothesis 11: We interpret the local contribution to the energy in different configurations of the spins as the volume occupied by grains in different local orientations. In modelling terms, its random structure is an obvious advantage in the context of the disordered nature of granular media: Additionally, the locally fluctuating connectivity may be thought of as modelling the range of coordination numbers of the grains 13.

This Hamiltonian has recently been studied on a random graph in the context of satisfiability problems in combinatorial optimization [14, and on a $2 \mathrm{D}$ triangular lattice [15]. It has a trivial ground state where all spins point up and all plaquettes are in the configuration +++ giving a contribution of -1 to the energy. Yet, locally, plaquettes of the type ,,--+-+-+-- (satisfied plaquettes) also give the same contribution, although one may not be able to cover the entire graph with these 4 types of plaquettes in equal proportions. In this case, which occurs for $c>c_{c} \sim 2.75$ [14] most ground states will be ferromagnetic corresponding to a state with long-range order and a crystalline state of the granular medium; reaching the state +++ from,,--+-+-+-- requires two spin-flips and a crossing of an energetic barrier.

We argue that the 3-spins ferromagnetic Hamiltonian is a suitable model-Hamiltonian for two reasons: The mechanism by which energy barrier has to be crossed in going from one metastable state to another aims to model the situation where a grain in order to reach a void in a granular assembly has to push apart temporarily other grains thus increasing the volume. This has recently been argued to be an important ingredient in models of granular compaction 16].

The feature of the model responsible for the slow dynamics, however, is the degeneracy of the four configurations of plaquettes with $s_{i} s_{j} s_{k}=1$ resulting in competition between satisfying plaquettes (dense arrangements of particles) locally (all states with even parity may be used, resulting in a large entropy) and global order (only the +++ state may be used). When grains are shaken, they rearrange locally, but locally dense configurations can be mutually incompatible: voids appear between densely packed clusters due to mutually incompatible grain orientations (geometric frustration) - leading to competition between locally dense packings and global crystalline order.

To mimic the action of tapping leading to compaction we choose the following dynamics: Each tap consists of two phases. First, in the dilation phase, a small fraction (taken to be $10^{-4}$ ) of the sites is chosen at random and have their spins flipped. This corresponds to the 


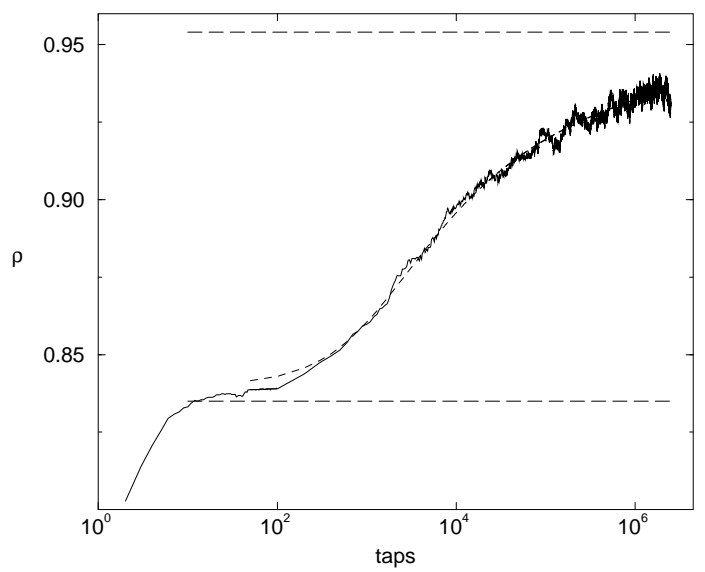

Fig. 1 - Compaction curve at connectivity $c=3$ for a system of $10^{4}$ spins (one spin is flipped at random per tap). The data stem from a single run with random initial conditions and the fit (dashed line) follows (2) with parameters $\rho_{\infty}=.971, \rho_{0}=.840, D=2.76$, and $\tau=1510$. The long-dashed line (top) indicates the approximate density 0.954 at which the dynamical transition occurs, the longdashed line (bottom) indicates the approximate density 0.835 at which the fast dynamics stops, the single-particle relaxation threshold.

rapid acceleration of the sample during tapping. Then, the system undergoes several (in our case 3) Monte-Carlo sweeps at zero temperature: Each sweep consists of choosing $N$ sites in series and flipping their spins with probability 1 if the flip lowers the energy, with probability $1 / 2$ if the flip leaves the energy unchanged. This phase corresponds to the relaxation process after the tap, where the particles reach a new locally stable configuration.

This dynamics is a simplified version, suitable for spin models, of the tapping dynamics used in cooperative Monte Carlo simulations of sphere shaking [17]. It has been recently introduced independently in the context of spins models of granular compaction by Dean and Lefèvre [18] (however their 2-spin models lack the competition between local and global order). In the context of combinatorial optimization it corresponds to the Walksat-algorithm, known to be one of the most efficient algorithms for the solution of satisfiability problems [19].

An example of a single run of the system defined by (11) is shown in Figure 1. We can identify three regimes of this dynamics, first a very fast increase of the density up to a density $\rho_{0}$, then the slow compaction regime which takes the density up to $\rho_{\infty}$, and finally an asymptotic regime.

In the first regime, which only lasts a few taps, all sites orient their spins in parallel with the local field acting on that site. This corresponds to a fast dynamics whereby single particles locally find the orientation maximizing the density leading to the density of $\rho_{0}\left({ }^{1}\right)$. We therefore term this the single-particle relaxation threshold (SPRT).

This fast regime may be illustrated by considering a single site $i$ connected to $2 k_{i}$ other sites and subject to the local field $h_{i}=1 / 2 \sum_{j k} C_{i j k} s_{j} s_{k}$. For random initial conditions, the values of $l_{i}=h_{i} s_{i}$ are binomially distributed with a probability of $C_{\left(k_{i}-l_{i}\right) / 2}^{k_{i}}(1 / 2)^{k_{i}}$ if $k_{i}-l_{i}$ is even and zero if it is odd. If $l_{i}<0$ zero-temperature dynamics will flip this spin, turn $l_{i}$

$\left({ }^{1}\right)$ During the fast regime one may also model the mobility of the grains by a dynamics of the bonds subject to kinetic constraints; the resulting graph typically differs from those constructed according to (11), however the compaction dynamics does not change qualitatively. 
to $-l_{i}$ and turn $\left(k_{i} \pm l_{i}\right) / 2$ satisfied (dissatisfied) plaquettes connected to it into dissatisfied (satisfied) ones. This will cause the $l_{j}$ of $k_{i} \pm l_{i}$ neighbouring sites to decrease (increase) by 2. This dynamics stops when all sites have $l \geq 0$ giving $\rho_{0}=1 /(3 N) \sum_{i} l_{i}$.

Neglecting loops in the random graph, the fast dynamics may be modelled by a simple population dynamics of $N$ elements, each with a Poisson distributed value of $k_{i}$ and a value of $l_{i}$ distributed according to the initial binomial distribution. At each step a randomly chosen element with negative $l_{i}$ has its $l_{i}$ inverted, and $k_{i} \pm l_{i}$ randomly chosen elements have their values of $l$ decreased (increased) by 2 until $l_{i} \geq 0 \forall i$. Running the population dynamics for $N=10^{4}$ at $c=3$ we obtain $\rho_{0}=0.835(1)$ which is shown as a dotted line in Figure 1. Note that this density is found to be much higher than that of a typical 'blocked' state with $l_{i} \geq 0 \forall i$ which is found to be 0.49 [20](see also the discussion in [21]); despite the fact that these states are exponentially dominant, the non-equilibrium, non-ergodic nature of the fast dynamics is responsible for taking the system to an (atypical) blocked state of higher density. At the end of the fast dynamics, there are no sites with more frustrated than unfrustrated plaquettes connected to them. The SPRT density thus appears as the density which is reached dynamically by putting each particle into its locally optimal configuration, as has also been found in lattice-based models [8] and simulations of sphere packings [16], [24], which incorporate both fast and slow dynamics.

The second phase of the dynamics consists of removing some of the remaining frustrated plaquettes and gives a logarithmically slow compaction [2, 8, 24 leading from density $\rho_{0}$ to $\rho_{\infty}$. The compaction curve may be fitted to the well-known logarithmic law [2]

$$
\rho(t)=\rho_{\infty}-\left(\rho_{\infty}-\rho_{0}\right) /(1+1 / D \ln (1+t / \tau)),
$$

which may also be written in the simple form $1+t(\rho) / \tau=\exp \left\{D \frac{\rho-\rho_{0}}{\rho_{\infty}-\rho}\right\}$, implying that the dynamics becomes slow (logarithmic) as soon as the density reaches $\rho_{0}$. With increasing density, free-energy barriers rise up causing the dynamics to slow down according to (2). The point where the height of these barriers scales with the system size marks a breaking of the ergodicity of the dynamics, a break-up of the phase-space into a large number (scaling exponentially with the system size) of disconnected clusters, and a saturation of the compaction curve. For small driving amplitudes, we thus identify the asymptotic density (random close packing) with a dynamical phase transition [10,12, 25].

To support this picture we give a simple approximation for the density $\rho_{\infty}$ at which the dynamical transition occurs. Using the replica-trick $\ln Z=\lim _{n \rightarrow 0} \partial_{n} Z^{n}$ [26] and standard manipulations we obtain for the average of the $n$-th power of the partition-function of the Hamiltonian (1) averaged the ensemble of random graphs

$$
\begin{array}{r}
\left\langle\left\langle\quad Z^{n}\right\rangle\right\rangle=\prod_{\vec{\sigma}} \int_{0}^{1} d c(\vec{\sigma}) \exp \left\{-N\left(\sum_{\vec{\sigma}} c(\vec{\sigma}) \ln (c(\vec{\sigma}))\right.\right. \\
\left.\left.+c / 3-c / 3 \sum_{\vec{\omega}, \vec{\tau}, \vec{\sigma}} c(\vec{\omega}) c(\vec{\tau}) c(\vec{\sigma}) \exp \left\{\beta \sum_{a} \omega^{a} \tau^{a} \sigma^{a}\right\}\right)\right\},
\end{array}
$$

where $c(\vec{\sigma})$ is an order parameter function defined on the domain of the $2^{n}$ vectors $\sigma^{a}= \pm 1$. A simple variational ansatz 14,27,28 implementing one step of replica-symmetry breaking (RSB)

$$
c(\vec{\sigma})=\prod_{b=1}^{n / m}\left\{\frac{\int d h^{b} G_{\Delta}\left(h^{b}\right) e^{\beta h^{b} \sum_{a=(b-1) m+1}^{b m} \sigma^{a}}}{\int d h^{b} G_{\Delta}\left(h^{b}\right)\left[2 \cosh \left(\beta h^{b}\right)\right]^{m}}\right\},
$$




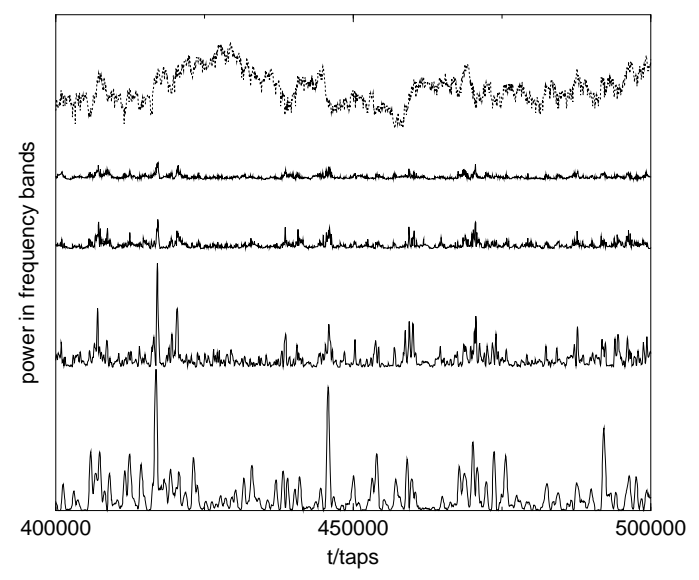

Fig. 2 - The Fourier-transform of the preceding 1024 taps is used to produce a power spectrum as a function of time. We plot the power in the first octave (frequency $1 /(1024$ taps) $-2 /(1024$ taps)), second octave (frequency $2 /(1024$ taps) - 4/(1024 taps)), etc. up to the fourth octave (bottom to top). One finds that the fluctuations of the power in the different frequency bands are strongly correlated across many octaves, and correspond to sudden changes in the density (shown as dotted line).

where $G_{\Delta}(h)$ is a Gaussian with zero mean and variance $\Delta$, gives the free energy subject to the variational ansatz $f(\beta)=\operatorname{extr}_{\Delta, m} f_{1}(\beta, \Delta, m)$ with

$$
\begin{aligned}
\beta \quad & f_{1}(\beta, \Delta, m)=\frac{\int D z(\beta \sqrt{\Delta} z)[2 \cosh (\beta \sqrt{\Delta} z)]^{m-1} \sinh (\beta \sqrt{\Delta} z)}{\int D z[2 \cosh (\beta \sqrt{\Delta} z)]^{m}} \\
& -\frac{1-c}{m} \ln \left(\int D z[2 \cosh (\beta \sqrt{\Delta} z)]^{m}\right)-c /(3 m) \ln \left(\iiint D z_{1} D z_{2} D z_{3}\right. \\
& {\left.\left[8 \cosh \left(\beta \sqrt{\Delta} z_{1}\right) \cosh \left(\beta \sqrt{\Delta} z_{2}\right) \cosh \left(\beta \sqrt{\Delta} z_{3}\right) \cosh (\beta)+8 \sinh \left(\beta \sqrt{\Delta} z_{1}\right) \sinh \left(\beta \sqrt{\Delta} z_{2}\right) \sinh \left(\beta \sqrt{\Delta} z_{3}\right) \sinh (\beta)\right]^{m}\right), }
\end{aligned}
$$

where $D(z)$ denotes the Gaussian measure with zero mean and variance one. The dynamical transition occurs at a temperature where $\partial(\beta f(\beta, \Delta, m)) / \partial m$ evaluated at $m=1$ develops a minimum at finite $\Delta[25]$. The corresponding density is marked with a horizontal line in Figure 1 and agrees well with the asymptotic density reached by the tapping dynamics.

The third regime of the compaction process is reached only asymptotically and consists of fluctuations around the random-close-packing density.

Figure 1 shows marked fluctuations around the logarithmic compaction law, which have been the subject of detailed experimental investigations [29], where marked correlations between the Fourier components of different frequencies of these fluctuations were found. In order to compare the results of our model with experiment we follow [29] and use Fourier transforms to plot the power of the timeseries $\rho(t)$ in different frequency bands against time in Figure 2. As in the experimental data, the fluctuation of the power in a given band shows marked 'bursts' after periods of calm. As a result of these bursts the fluctuations in the the power spectrum are correlated over a wide range of frequencies: In figure 3 we have plotted, as in [29], the average of the correlation matrix $C_{i j}$ as a function of octave separation $|i-j|$, where $C_{i j}:=M_{i j} \sqrt{\frac{M_{i i} M_{j j}}{\left(M_{i i}-1\right)\left(M_{j j}-1\right)}}$ probes the non-Gaussian components of the correlation of the noise power in the $i$ th and $j$ th octaves. $M_{i j}$ is defined as the covariances of noise power fluctuations $\left.\left\langle\delta O_{i} \delta O_{j}\right\rangle / \sqrt{\langle}\left(\delta O_{i}\right)^{2}\right\rangle\left\langle\left(\delta O_{j}\right)^{2}\right\rangle$ for $i \neq j$ and $M_{i i}:=\left\langle\left(\delta O_{j}\right)^{2}\right\rangle / \sum_{k \in i}\left\langle P_{k}\right\rangle^{2}$, where 


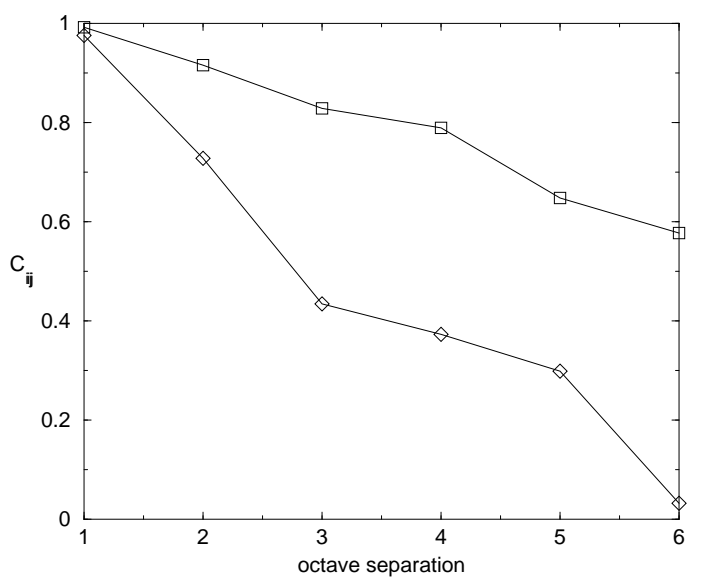

Fig. 3 - The rescaled covariances of the power fluctuations as are plotted as a function of the octave separation for both the ferromagnetic 3-spin model (squares) and the parking-lot model (diamonds). The definition of these quantities is provided in the text, a high value of the rescaled covariance indicates strong correlations of the power-fluctuations of two given frequency bands.

the average is over 5000 time steps in the asymptotic regime, $\delta O_{i}$ are the power fluctuation around the average in the $i$ th octave and $P_{k}, k \in i$ is the power in the $k$ th frequency bin in octave $i$. Figure 3 also shows the corresponding results for the parking-lot model [30], where particles of a certain size are desorbed from and absorbed by a surface at random, subject to the constraint that none of the particles overlap.

The experimental data analyzed in 29] shows a slow decay of the average $C_{i j}$ with octave separation (particularly in the bottom and top of the sample) with $C_{i j}$ decaying to about 0.6 over 6 decades. This behaviour is reproduced by the 3 -spin model, but not by the parkinglot model. We argue that the 'burst' found in the time-series of the density is resposible for the correlation of noise power over a wide range of frequencies. In spin models with finite connectivity such bursts arise quite naturally due to cascades of spin-flips. The crucial mechanism is that the flipping of a single spin alters the local fields acting on its neighbouring sites. The configuration of the spins on these sites may then no longer be locally stable, causing them to flip in turn. The first spin thus acts as a 'plug' releasing the neighbouring spins and setting off a cascade of successive spin-flips. A plug may also be composed of two or more sites, which need to have their spins flipped before neighbouring spins are released.

Note that the cascade mechanism is entirely absent not only from generic fully connected models (where each spin interacts with all spins in the system, but with an interaction energy scaling as $1 / \sqrt{N}$ ) but also from the parking-lot model: Clearly the creation and filling of such a gap by a particle does not cause the appearance of further gaps, in the way spins flips may trigger a cascade. For this reason, the bursts observed in experiment and in our model are absent in the parking-lot model as noted in [29]. In other, lattice-based models [4, \&] of finitely connected granular media, however, cascades are also to be expected.

To conclude, we have presented a finitely connected spin model of vibrated granular matter, which while reproducing the slow logarithmic relaxation associated with compaction, has also thrown new light on the fast relaxation mechanisms in granular dynamics. Within this model we have also identified the asymptotic density reached with a dynamical phase transition, and interpreted the density-noise power fluctuations observed in recent experiments in terms of 
spin flip cascades.

AM is very grateful to the ICTP Trieste for providing an extremely pleasant and stimulating environment where much of this work was carried out. We thank A. Barrat, S. Franz, M. Leone, E. Nowak, F. Ricci-Tersenghi, P. Stadler, M. Weigt, and R. Zecchina for illuminating discussions.

\section{REFERENCES}

[1] S. F. Edwards and A. Mehta, Journal de Physique 50, 2489 (1989).

[2] E. R. Nowak, J. Knight, E. Ben-Naim, H. Jaeger, and S. R. Nagel, Phys. Rev.E 57(2),1971 (1998).

[3] A. Mehta and G. C. Barker, Europhysics Letters 27, 501 (1994).

[4] A. Fierro, A. de Candia, and A. Coniglio, cond-mat/0009173 and references therein.

[5] W. Kob and H.C. Andersen, Phys. Rev. E 48, 4364 (1993)

[6] G. Biroli and M. Mézard cond-mat/0106309

[7] J. D. Shore, M. Holzer, and J. P. Sethna, Phys. Rev. B46, 11376 (1992)

[8] P. F. Stadler, J. M. Luck and A. Mehta, cond-mat/0103076.

[9] J. Kurchan, J. Phys. Cond. Mat, 12, 6611 (2000).

[10] L. Berthier, L.F. Cugliandolo, J. L. Iguain, condmat/0010266.

[11] S. F. Edwards, in Granular Matter: An Interdisciplinary Approach, ed. A. Mehta, (SpringerVerlag, New York, 1994).

[12] Models with $p$-spin interactions $(p>2)$ have been used extensively as models of structural glasses, for a review see J.-P. Bouchaud, L.F. Cugliandolo, J. Kurchan, and M. Mezard, in "Spin Glasses and Random fields", A.P. Young ed., World Scientific (1997).

[13] G. C. Barker and A. Mehta, Physical Review A45, 3435 (1992).

[14] F. Ricci-Tersenghi, M. Weigt, and R. Zecchina, Phys. Rev. E 63, 026702 (2001).

[15] M. Newman and C. Moore, Phys Rev. E60(5),5068 (1999).

[16] A. Mehta and G C Barker, J. Phys - Cond. Mat., 12, 6619-6628, (2000).

[17] A. Mehta and G C Barker, Physical Review Letters 67, 394 (1991).

[18] D. S. Dean and A. Lefèvre, Phys. Rev. Lett. 86, 5639 (2001), cond-mat/0101166, A. Lefèvre and D. S. Dean, cond-mat/0102204, D. S. Dean and A. Lefèvre,cond-mat/0106220.

[19] B.Selman, H.A. Kautz and B. Cohen in "Cliques, Coloring, and Satisfiability: Second DIMACS Implementation Challenge, October 11-13, 1993", D.S. Johnson and M.A. Trick eds., DIMACS Series in Discrete Mathematics and Theoretical Computer Science, vol. 26, AMS, (1996).

[20] J. Berg and A. Mehta, unpublished.

[21] A. Barrat, J. Kurchan,V. Loreto, and M. Sellitto, Phys. Rev. Lett.85,5034 (2000); A. Barrat, J. Kurchan, V. Loreto and M. Sellitto, cond-mat/0011492.

[22] A. Barrat and R. Zecchina, Phys. Rev. E 59 R1299 (1999).

[23] E. R. Weeks, J. C. Crocker, A. C. Levitt, A. Schofield and D. A. Weitz, Science 287, 627.

[24] G. C. Barker and A. Mehta, Physical Review E47, 184 (1993); G. C. Barker and A. Mehta, cond-mat/0010268.

[25] R. Monasson, Phys. Rev. Lett.75,2847 (1995), S. Franz, G. Parisi, J. Physique I5,1401 (1995).

[26] Mézard,M., Parisi,G., and Virasoro, M. A. Spin Glass Theory and Beyond, World Scientific (Singapore, 1987).

[27] G. Biroli, R. Monasson, and M. Weigt, Eur. Phys. J. B 14, 551 (2000)

[28] S. Franz, M. Mezard, F. Ricci-Tersenghi, M. Weigt and R. Zecchina, cond-mat/0103026

[29] E.R. Nowak, A. Grushin, A. Barnum, and M. Weissman, Phys. Rev.E 63, 020301 (2001)

[30] A. J. Kolan, E. R. Nowak and A. V. Tchakenko, Phys. Rev. E 593094 (1999). 